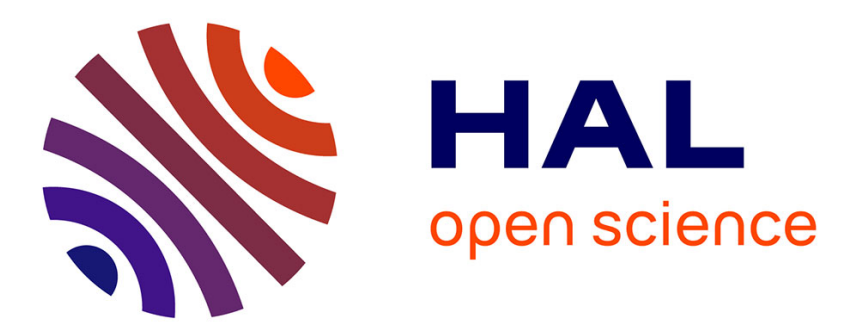

\title{
Quartz Resonator Instabilities Under Cryogenic Conditions
}

Maxim Goryachev, Serge Galliou, Philippe Abbé, Pierre-Yves Bourgeois, Serge Grop, Benoit Dubois

\section{To cite this version:}

Maxim Goryachev, Serge Galliou, Philippe Abbé, Pierre-Yves Bourgeois, Serge Grop, et al.. Quartz Resonator Instabilities Under Cryogenic Conditions. IEEE Transactions on Ultrasonics, Ferroelectrics and Frequency Control, 2012, 59 (1), pp.21-29. 10.1109/TUFFC.2012.2152 . hal-00690659

\section{HAL Id: hal-00690659 \\ https://hal.science/hal-00690659}

Submitted on 5 May 2021

HAL is a multi-disciplinary open access archive for the deposit and dissemination of scientific research documents, whether they are published or not. The documents may come from teaching and research institutions in France or abroad, or from public or private research centers.
L'archive ouverte pluridisciplinaire HAL, est destinée au dépôt et à la diffusion de documents scientifiques de niveau recherche, publiés ou non, émanant des établissements d'enseignement et de recherche français ou étrangers, des laboratoires publics ou privés.

\section{(c)(1)}

Distributed under a Creative Commons Attribution| 4.0 International License 


\title{
Quartz Resonator Instabilities Under Cryogenic Conditions
}

\author{
Maxim Goryachev, Serge Galliou, Philippe Abbé, Pierre-Yves Bourgeois, Serge Grop, and Benoît Dubois
}

\begin{abstract}
The phase noise of a quartz crystal resonator working at liquid helium temperatures is studied. Measurement methods and the device environment are explained. The phase noise is measured for different resonance modes, excitation levels, amount of operating time, device orientations in relation to the cryocooler vibration axis, and temperatures. Stability limits of a frequency source based on such devices are evaluated in the present measurement conditions. The sources of phase flicker and white noises are identified. Finally, the results are compared with previous works.
\end{abstract}

\section{INTRODUCTION}

Since the dawn of the cryogenic era in the 1950s, it Shas been widely known that quartz crystal BAW resonators exhibit very high quality factors at liquid helium temperatures [1]-[4]. Impressed by these results, later researchers revived the idea of investigating BAW resonators at cryogenic temperatures, achieving even higher values [5]-[9]. All these works are nonsystematic studies of some particular aspects of BAW device operation. For example, only some of the works note frequency-temperature resonator characteristics which are very important to device performance. Furthermore, no studies were done concerning resonator nonlinearities, different mode comparison, or analysis of loss phenomena. Some studies were partially related to quartz resonator proper phase noise at liquid helium temperatures [7], [10], [11], but their results are questionable and are discussed in this work. The nonsystematic approach of these studies is a consequence of the many difficulties associated with cryogenic experiments. Indeed, the first investigations on the high quality factor resonators at cryogenic temperatures were made with bath cryostats. These systems have several disadvantages, such as difficulties of operation and experiment time limitations. In addition, experiment time limitations made long frequency stability measurements almost impossible. Recently, cryocoolers became widely available for liquid helium temperature experiments. These devices do not have any limitation on measurement time and they are rather simple in operation. These provided new opportunities to revive this area of research. The idea of

The authors are with the Time and Frequency Department of the Franche-Comté Electronique, Mécanique, Thermique et Optique-Sciences et Technologies (FEMTO-ST) Institute, Besançon, France (e-mail: maxim.goryachev@femto-st.fr). practical use of cryogenic quartz resonators has become especially important in the light of quartz technology perspectives in general. Therefore, since 2007, some systematic researches in the field have been conducted at the Franche-Comté Electronique, Mécanique, Thermique et Optique-Sciences et Technologies (FEMTO-ST) Institute, Besançon, France. Thus, the different operation modes have been studied and compared, paying attention to frequency-temperature characteristics [12], [13]. Based on measurement data, the loss phenomena in quartz resonators were analyzed [14]. Further, amplitude-frequency nonlinear effects, temperature sensitivity, LGT resonator investigation, and mode comparison [15] are also subjects of this research. The present work is a continuation of this global investigation. It concerns quartz resonator instabilities at cryogenic conditions.

The present research deals with quartz botier à vieillissement amélioré (BVA) resonators [16], because it has been already shown that electrodeless resonators exhibit higher quality factors than electrode-deposited resonators [6]. More precisely, measurements are made with planoconvex SC-cut resonators [17] optimized to work on the $3 \mathrm{rd}$ overtone of the $\mathrm{C}$-mode $(5 \mathrm{MHz})$ at $80^{\circ} \mathrm{C}$. At this temperature, the frequency-temperature characteristic of such a device exhibits a turn-over point. The devices are state-of-the-art commercial resonators provided by the BVA Industrie Company (Besançon, France).

The paper starts with the description of the cryogenic system and the main sources of environmental disturbance (Section II), and then passes to explanations of the phase noise measurement method in cryogenic conditions and preliminary results (Section III) for the open-loop case. Section IV considers resonator long-term instabilities measured in closed loop. Section V presents the resonator phase noise as a function of different parameters. In the final section, the obtained results are compared with some earlier works.

\section{Measurement Setup}

In this work, a two-stage pulse-tube cryocooler (see Fig. 1) is used at the cryogenic system's core. The cryocooler is shielded with two protection screens: a vacuum chamber and an anti-radiation shield. The former prevents temperature losses resulting from heat conduction through air. The air pressure inside the chamber is about $10^{-5} \mathrm{~Pa}$. This shield is at room temperature and is made with a light-reflecting material. Both primary and secondary pumps are cut off from the chamber during the 


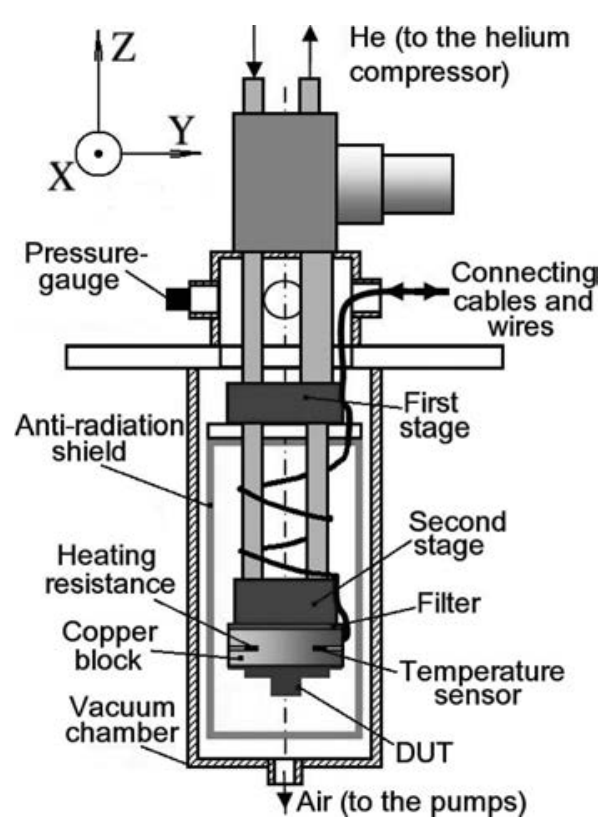

Fig. 1. Cryogenic system.

whole measurement time. The anti-radiation shield is attached to the first cryocooler stage and its temperature is about 50K. Its inner and outer surfaces are covered with a specially fabricated light-reflecting paper comprising several layers of metalized mylar sheeting to reduce radiation losses of the second stage.

Nominally, the cryocooler absorbs up to $1 \mathrm{~W}$ at its second stage at $4 \mathrm{~K}$. The second stage temperature can go down to almost $3 \mathrm{~K}$ in the unloaded configuration. The thermal losses are mainly introduced with connecting cables needed for measurements and environmental control. The device under test (DUT) is attached to a temperature-controlled copper block.

The almost periodic temperature fluctuations of the cryocooler second stage resulting from the helium pulsation are about $0.3 \mathrm{~K}$. The power spectral density (PSD) of temperature fluctuations of the second stage in the steady state consists of an $f^{-2}$ region for frequencies less than about $10^{-2} \mathrm{~Hz}$ and an $f^{0}$ region for higher frequencies. In addition, a clear spurious frequency of $1.7 \mathrm{~Hz}$ and its harmonics are present as a result of temperature pulsations initiated by the pulse tube cycle.

To improve the thermal environment of the DUT, passive and active temperature stabilization methods are used. First, a thermal filter is introduced between the second stage and the copper block (see Fig. 1). The filter reduces the temperature pulsation down to $15 \mathrm{mK}$, but increases the absolute temperature difference between both parts. This difference mainly depends on the number of cables connected to the DUT. Second, the active temperature regulation is implemented with a temperature controller (model 332, Lake Shore Cryotronics Inc., Westerville, $\mathrm{OH}$ ), a Cernox temperature sensor (Lake Shore Cryotronics Inc.), and a $20-\Omega$ heating resistor. The sensor and the actuator are installed inside the copper block under the filter. The control system eliminates the $f^{-2}$ region in the

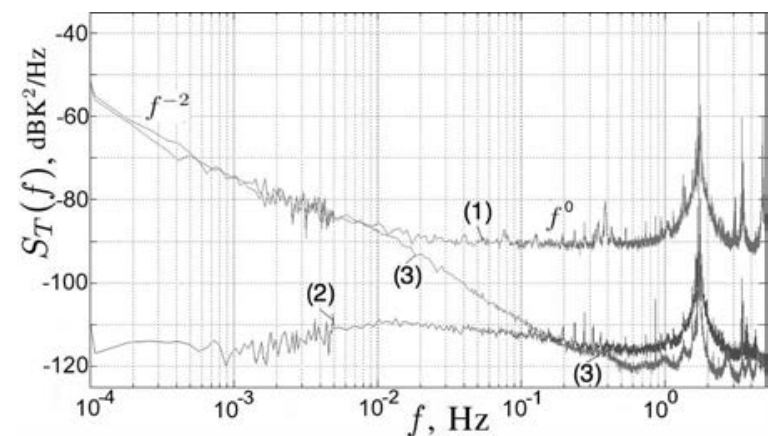

Fig. 2. Power spectral density of temperature fluctuations: (1) at the cryocooler second stage, (2) at the device under test (DUT) level with the active temperature control, (3) at the DUT level without the temperature control.

temperature fluctuation spectrum (for frequencies higher than $10^{-4} \mathrm{~Hz}$ ) and reduces the temperature pulsation to $5 \mathrm{mK}$. The power spectral densities of temperature fluctuations in both second stage (unregulated and non-filtrated part) and the DUT (after filtering with and without active regulation) is shown in Fig. 2.

Fig. 2 shows that the remaining noise resulting from temperature instabilities at the DUT level (with no thermal load or cables installed) is almost a white noise in the frequency range of $10^{-3}$ to $10^{0} \mathrm{~Hz}$. Although it must be noted that the $f^{-2}$ spectrum slope can still appear at this range of frequencies at the DUT level when measurements are performed during exponential transients in the whole thermo-mechanical system (cryogenerator, cables, DUT, etc.). This happens when the thermal load is high enough and the connection cables thermally relate regulated and unregulated parts of the system.

Another type of cryocooler environmental disturbance is vibration. According to the cryocooler specification, the permanent pulsation of helium inside the pulse-tube causes a DUT vibration with an amplitude $A=10 \mu \mathrm{m}$ and a frequency $f=1.7 \mathrm{~Hz}$. The spectrum of vertical DUT acceleration ( $Z$-axis in Fig. 1) has been calculated, based on measurements made with a high-sensitivity piezoelectric accelerometer $\mathrm{A} / 600$ and a high-speed real-time system ADwin-Pro II (Jäger Computergesteuerte Messtechnik GmbH, Lorsch, Germany) with a custom-made low-noise charge amplifier. The DUT acceleration PSD along the vertical axis is shown in Fig. 3. In fact, the sensitivity of the accelerometer at cryogenic temperature is unknown, so the presented data lacks an accurately calibrated scale.

Nevertheless, the measurement suggests that the DUT is subject to $f^{-1}$ instabilities in the frequency range of $10^{-2}$ to $10^{1} \mathrm{~Hz}$ resulting from external vibration in the vertical axis. It must be noted that no vibration compensation system is installed for the presented measurements.

\section{Open-Loop Measurements}

Usually, at room temperatures the phase noise of ultrastable quartz crystal resonators is measured with an inter- 


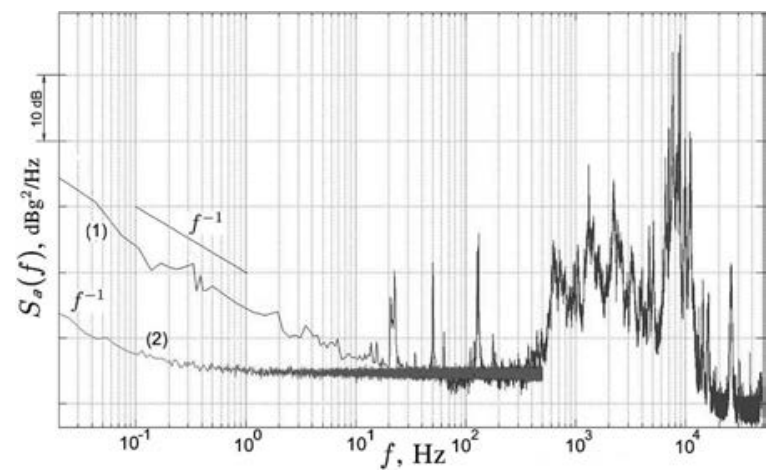

Fig. 3. Device under test (DUT) acceleration spectrum (vertical axis only): (1) DUT acceleration at 7K (not calibrated), (2) charge amplifier noise floor.

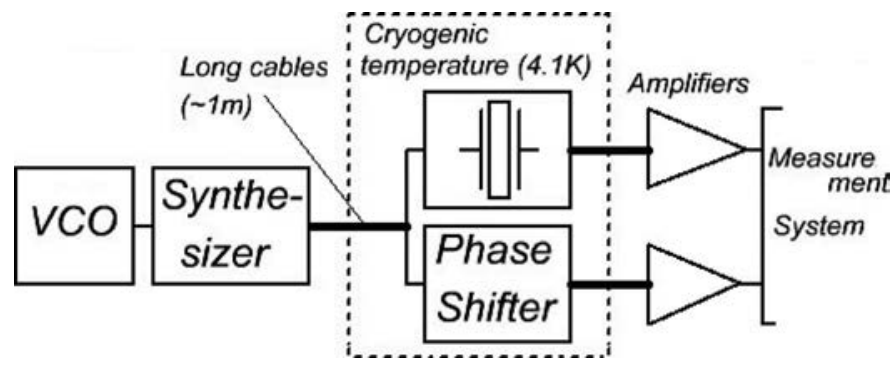

Fig. 4. Open-loop measurement scheme for cryogenic resonator instabilities.

ferometric measurement system [18]. Such a scheme is not possible for resonators working under cryogenic conditions because of a significant dispersion of resonator parameters at low temperatures. Thus, for example, two $5-\mathrm{MHz}$ resonators (from the same batch) can exhibit a resonance frequency difference of $1.1 \mathrm{kHz}$, for the 5 th overtone of the A-mode, at $4.2 \mathrm{~K}$. For $10-\mathrm{MHz}$ resonators, this difference is about $500 \mathrm{~Hz}$. This phenomenon is due to small differences in crystal blank orientations [10]. This difference cannot be covered with trimming capacitors as in the case of room-temperature measurements. Therefore, to evaluate the resonator phase noise under cryocooler conditions, only a one-resonator scheme is available (see Fig. 4). Note that the synthesizer is an RF signal generator HP8665B (Agilent Technologies Inc., Santa Clara, CA). It is used to tune an excitation signal frequency to the resonance frequency.

Measurements are made step-by-step to identify and characterize all noise sources of the measurement system and to assure that the noise floor is lower than the measured noise. All the phase noise or frequency stability measurements are made with a phase noise test set (TSC5120A, Symmetricom Corp., San Jose, CA) which uses a cross correlation technique.

At the first step, the measurement noise floor is evaluated. To do so, the signal from a voltage-controlled oscillator ( $\mathrm{VCO}$ ) is split and used as the measured signal and as the reference of the phase noise test set. The result is shown in Fig. 5, curve (1).

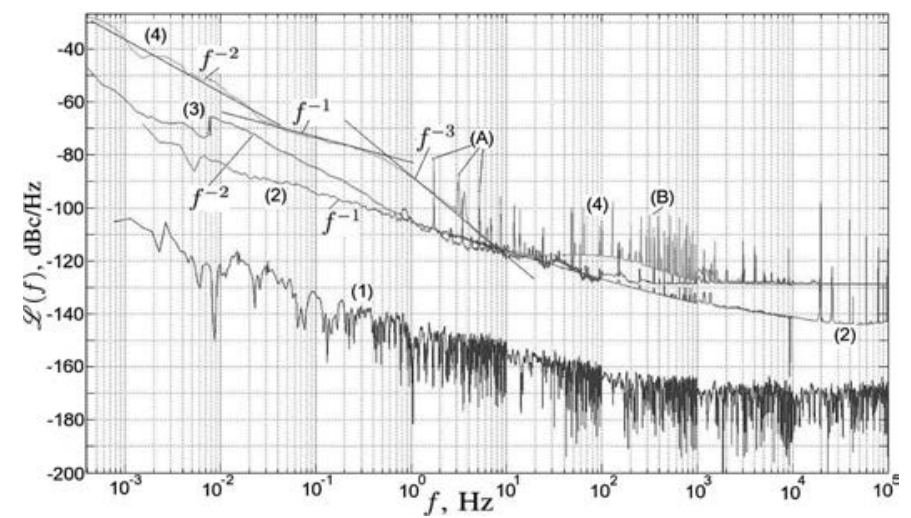

Fig. 5. Phase noise spectra of different measurement experiments: (1) measurement system noise floor, (2) amplifier phase noise, (3) phase noise of the cryogenic assembly without the resonator, (4) phase noise of the cryogenic part with the resonator. (A) deterministic instabilities caused by the cryogenerator $(1.7 \mathrm{~Hz}$ and its harmonics), (B) synthesizer phase noise.

Second, the phase noise of two amplifiers is measured by adding these devices into each branch of the system [see curve (2) for the result].

Third, the cryogenic assembly, except for the resonator, is added to the measurement scheme. The cryogenic assembly includes the connectors, cables, and passive electronic components. At this step, the resonator is replaced with a resistance corresponding to its equivalent resistance at the cryogenic temperature. This measurement gives an estimation of the total noise floor of the cryogenic phase noise test bench. The result is presented in Fig. 5, curve (3).

Fourth, the measurements are repeated the same scheme as in the third step, but with the crystal resonator inside its $\pi$-network. The frequency of the signal at the output of the synthesizer must be adjusted exactly to the resonant frequency of the crystal device at the working temperature. In Fig. 5, curve (4) shows the results of these measurements for the 5 th overtone of the A-mode [15]. This mode has the highest value of quality factor $(Q$ $=325 \cdot 10^{6}$ at $15.6 \mathrm{MHz}$ ) compared with that of the third and fifth overtones. Therefore, it is the best candidate for further closed-loop frequency measurements. In addition, the 5 th overtone of the B mode $\left(Q=50.5 \cdot 10^{6}\right.$ at $9.2 \mathrm{MHz})$ and the $3 \mathrm{rd}$ overtone of the A mode $(Q=114.6$ $\cdot 10^{6}$ at $9.4 \mathrm{MHz}$ ) are considered further.

The design of the cryogenic part of the measurement system implies several requirements. First, the resonator's loaded quality factor must be preserved. To do so, the load must be of the same order of magnitude as the resonator's motional resistance. Unfortunately, the equivalent motional resistance of the resonator at these modes and overtones is very low (approximately $3.5 \Omega$ for the 5 th overtone of the A-mode). Second, the influence of the connecting cables must be minimized. This also means that the loading resistances of the $\pi$-network must be minimized. Third, the resonator's nonlinear effects must be avoided to prevent the excess noise [19]. Indeed, a quartz 
crystal resonator at the cryogenic temperature is subject to a strong amplitude-frequency effect [15]. This mainly results from high values of the $Q$-factor, because the frequency shift is proportional to the product of $Q$ and the input signal power [20]. To limit this effect, the resonator active power must be kept low. This could be done by injecting lower-power signals or by increasing the resonator load. And, fourth, it is absolutely necessary that the $\pi$-network is able to provide at least $+7 \mathrm{dBm}$ at the output of the amplifiers, whose gain is limited. This could be achieved by increasing the level of the excitation signal or by increasing the resonator load. As can be seen, the four requirements are contradictory and difficult to fulfill simultaneously, so a trade-off must be achieved. This has been done by providing approximately $12 \%$ of the unloaded quality factor.

A crystal resonator can be differently installed inside the copper block of the cryogenerator. Various orientations of the crystal resonator in the copper block of the cryocooler second stage are possible. Indeed, the resonator plate can coincide with the $X-Y$ plane or with the $X-Z$ plane as shown in Fig. 1. All the measurements demonstrated in the present section are made with the crystal plate normal to the $Z$-axis.

The measurement system noise floor clearly consists of white and $f^{-1}$ noises [Fig. 5, curve (1)]. It is lower than all measurements in the present work. The amplifier noise power spectral density also exhibits the same noise slopes [see curve (2)]. Although these wide-band amplifiers are specified to be low-noise devices, their phase noise is relatively high. If one compares amplifier phase noise with that of the cryogenic assembly without the resonator [curve (3)], the random-walk phase noise appears for the low Fourier frequencies. Its origin is attributed to temperature fluctuations (it is known that temperature fluctuations are random walks whose spectral density is proportional to $f^{-2}$ ) of the long cables used to connect both cryogenic and ambient temperature parts. As previously mentioned, these cables are not temperature regulated and connect ambient temperature devices (at about 295K) and the cryogenic circuit (at $4.1 \mathrm{~K}$ ), with an intermediate thermal connection at the first stage of the cryogenerator (at about 50K). This type of noise becomes important mostly in the case in which long thermal transients of the system are not over. This is explained in Section V.

When comparing measurements of the cryogenic part with and without the resonator [curves (4) and (3), respectively, in Fig. 5], two observations can be made. First, inside the resonator bandwidth (Fourier frequencies lower than $0.5 \mathrm{~Hz}$ ), the $\mathrm{VCO}$ and synthesizer phase noises are compensated because both branches are similar, resulting from the fact that the resonator works as a flywheel. Therefore, in this first part of the spectrum, one obtains the sum of phase noises of the amplifiers, cables, and resonator. Because spectrum (4) in this region is considerably higher than spectrum (3) which does not contain the resonator phase noise, the difference can be attributed to the resonator phase noise. $f^{-2}$ and $f^{-1}$ slopes are present in this part of the PSD. Second, outside the resonator bandwidth, oscillator and synthesizer noises are no longer compensated, because in one of the branches the phase noise is filtered. In that case, the resulting phase noise includes noises from the amplifiers, cables, the oscillator, the synthesizer, and the resonator. In the region between 0.5 and $10 \mathrm{~Hz}$, the resonator phase noise is dominant. An $f^{-3}$ slope is clearly visible here. Between 10 and $200 \mathrm{~Hz}$ [zone (B) in Fig. 5], the synthesizer phase noise is the highest one (these values correspond to the synthesizer documentation). For Fourier frequencies higher than $200 \mathrm{~Hz}$, the cryogenic assembly noise overlaps the others. Thus, between the lowest possible Fourier frequency and $10 \mathrm{~Hz}$, one obtains the resonator phase noise. It must be noted that the presented measurements are completely realistic, because this phase noise is relatively high.

The measured quartz crystal resonator working at cryogenic conditions has a PSD consisting of $f^{-2}, f^{-1}$, and $f^{-3}$ slopes. These results are similar to measurements made at room temperature [21]. In fact, the resonator can be considered as a low-pass filter in the phase domain. Thus, the resonator filters its own phase noise, forming the $f^{-1}$ slope inside its bandwidth and the $f^{-3}$ slope outside; i.e., the output phase noise is the resonator flicker noise multiplied by a square of the resonator transfer function in the phase domain. The bandwidth of this filter corresponds to the corner frequency of the resonator phase noise PSD and is inversely proportional to the loaded quality factor. According to the measurement results, in the phase domain, the bandwidth of the resonator working at $15597319 \mathrm{~Hz}$ is about $0.5 \mathrm{~Hz}$, giving a loaded quality factor of about $Q_{1}=15.6 \cdot 10^{6}$. In addition to stochastic instabilities of the resonator, deterministic fluctuations can be observed. These periodic fluctuations at $1.7 \mathrm{~Hz}$ and its integer multiples [see peaks (A) in Fig. 5] are due to the cryogenerator working cycle, which produces temperature changes and vibrations.

It is interesting to get an idea of the stability achievable by an oscillator built on such a resonator working at liquid-helium temperatures with the same loaded $Q$ factor used previously. It is still assumed that the resonator flicker noise dominates within the device bandwidth in comparison with the noise of sustaining electronics. Thus, the Allan deviation floor of such an oscillator can be estimated as

$$
\sigma_{y}=\sqrt{2 \ln (2) h_{-1}}
$$

where $h_{-1}$ is the coefficient of the flicker power-law spectral density in terms of fractional frequency fluctuations (i.e., $\left.S_{y}(f)=h_{-1} f^{-1}\right)[22]$. For the resonator, the relationship between phase and frequency spectra in its bandwidth is

$$
S_{y}(f)=\left(\frac{1}{2 Q_{l}}\right)^{2} \cdot S_{\phi}(f)=\left(\frac{1}{2 Q_{l}}\right)^{2} \cdot 2 \mathcal{L}(f) .
$$

Thus, the power law coefficient $h_{-1}$ can be estimated from the value of the $f^{-1}$ asymptote line of $\mathcal{L}(f)$ at $f=1 \mathrm{~Hz}$ 


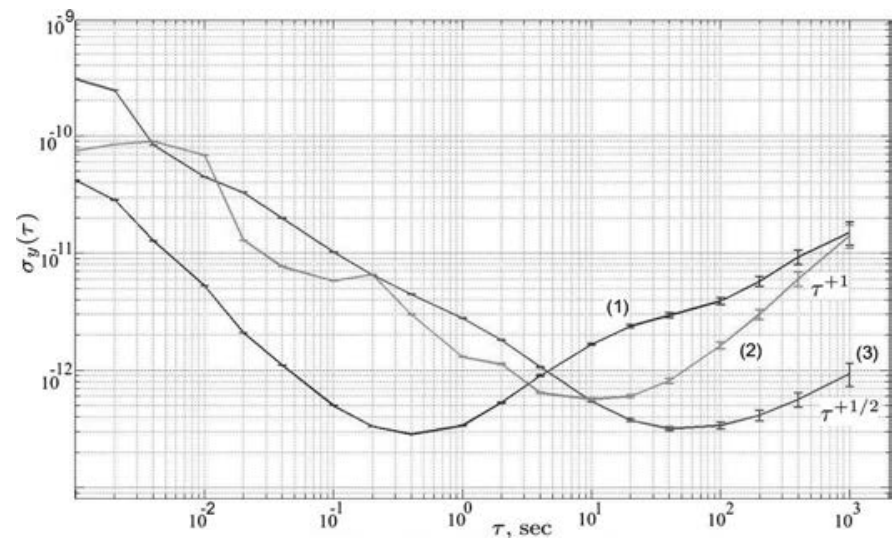

Fig. 6. Allan deviation of the closed-loop frequency stabilization system locked on the A-mode of 5th overtone measured (2) within the first 12 hours of resonator operation inside the cryocooler, (3) measured after at least 60 hours of resonator permanent operation in cryogenic environment, and compared with the stand-alone oscillator (1), i.e., the voltagecontrolled oscillator.

from curve (4) in Fig. 5. At $1 \mathrm{~Hz}$, this assymptote value is about $-83 \mathrm{~dB}$, giving $h_{-1}=1 \cdot 10^{-23}$. Consequently, the Allan deviation floor of such an oscillator is $\sigma_{y}=3.8$. $10^{-12}$.

This value is more than 10 times higher than that of actual state-of-the-art quartz crystal oscillators working at ambient temperatures. The origins of such a high phase noise are unknown. Three possible reasons could be identified: 1) cryogenerator-induced vibration, 2) temperature instability, and 3) intrinsic resonator noise. These reasons are discussed in Section V.

\section{Closed-Loop Measurements}

Some more important information on the cryogenic resonator instabilities can also be deduced when the VCO (see Fig. 4) is locked on the cryogenic resonator frequency [15]. To do so, the output signals of both amplifiers are applied to a double balanced mixer for a downconversion to dc voltage. After low-frequency filtering, the dc signal is applied back to the VCO to lock the loop. It can be clearly shown that the Allan deviation curve of such a feedback system may be separated into three regions (see Fig. 6). The first region, for averaging times $\tau<0.1 \mathrm{~s}$, is determined mainly by short-term instabilities coming from the VCO electronics and the cryogenic assembly. The second region $(0.1<\tau<10 \mathrm{~s})$ is due to noise sources of the loop elements. For the third region, $\tau>10 \mathrm{~s}$, the system Allan deviation level is proportional to the resonator noise and inversely proportional to its quality factor. By analyzing this last part, the resonator's long-term behavior can be estimated. This estimate is difficult to make using the open-loop approach.

Stability measurements shown in Fig. 6 are for the closed-loop system locked on the 5th overtone of the Amode. In these measurements, the frequency stability has been examined as a function of the amount of time of per- manent operation of the resonator inside the cryocooler. Therefore, Fig. 6 presents the Allan deviation measured during the first 12 hours [curve (2)] and after at least 60 hours of permanent operation [curve (3)]. Both results can be compared with the stand-alone oscillator stability [curve (1)]. As expected, the closed-loop degrades the system frequency stability for small averaging times, $\tau$, but improves it for large averaging times. For large $\tau$, curve (3) exhibits the same slope as the stand-alone oscillator, i.e., $\tau^{+1 / 2}$, which corresponds to the resonator $f^{-2}$ phase noise (phase random-walk). This could be shown analytically using linearized models in the domain of signal phase fluctuations [22]. On the other hand, after just $12 \mathrm{~h}$ of operation, the system shows a $\tau^{+1}$ law, i.e., a deterministic linear frequency drift. This drift probably results from a rapid aging of the resonator in the first hours after the cooling process, which changes its mechanical properties.

\section{Quartz Resonator Phase Noise at Cryogenic TEMPERATURES}

Section III explained the measurement techniques and gave phase noise measurement results for one usable system configuration. In this section, these measurements are extended to different resonance modes, excitation levels, amount of cryogenerator operation time, and crystal orientations. The main difference in the measurement technique introduced in this section is that neither branch of the cryogenic part is designed to work in the closed-loop system. First, this implies that the system can work with a lower driving signal. Second, both branches are designed to be identical except for the resonator in one branch and a resistance in the other branch. This is done to minimize the influence of external disturbances on the cryogenic assembly and, thus, to minimize the measurement noise floor.

First, one compares the results of the phase noise measured for different operating modes and overtones. Three modes are of interest here because of their relatively large quality factors: the 5 th overtone of the A-mode [curve (2) in Fig. 7], the 3rd overtone of the A-mode [curve (3), Fig. 7], and the 5th overtone of the B-mode [curve (4), Fig. 7]. It is clear from the figure that all three modes have very similar phase noise spectra. However, an $f^{-1}$ spectrum slope is almost absent for the 3rd overtone of the A-mode and the 5 th overtone of the B-mode overtone. This may be a consequence of their high sensitivity to temperature fluctuations [15], and thus a rather high $f^{-2}$ noise.

The next experiment compares phase noise spectra measured for the system with its resonator working at different excitation levels. Two measurements are made in this case. The first measurement is obtained with a level sufficient to provide $+7 \mathrm{dBm}$ at the inputs of the double balance mixer. Here, a $+0.5 \mathrm{dBm}$ level of the synthesizer output signal is used [see Fig. 8, curve (2)]. Another experiment is done with a much lower level of the synthesizer signal, i.e., $-4.5 \mathrm{dBm}$ [see Fig. 8, curve (3)]. The 


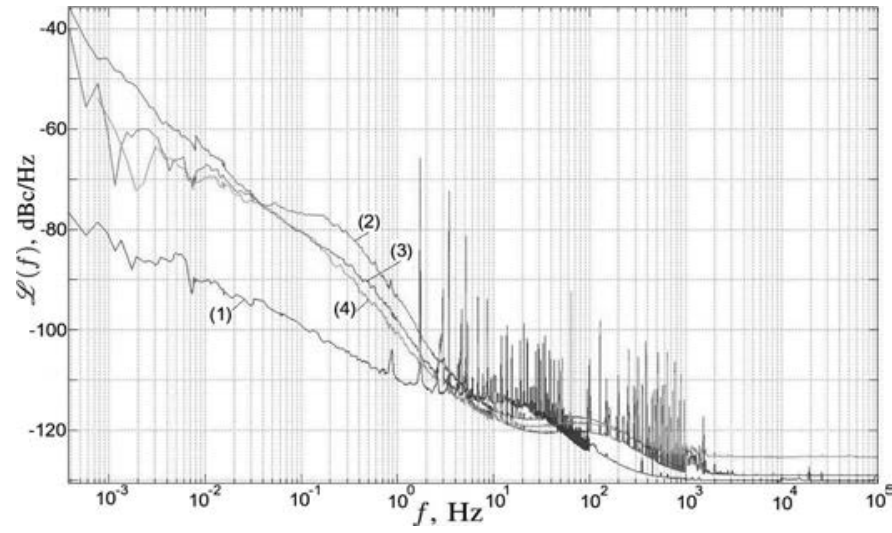

Fig. 7. Phase noise power spectral densities: (1) measurement phase noise floor, (2) cryogenic part phase noise for the 5th overtone of the Amode, (3) cryogenic part phase noise for the 3rd overtone of the A-mode, (4) cryogenic part phase noise for the 5th overtone of the B-mode.

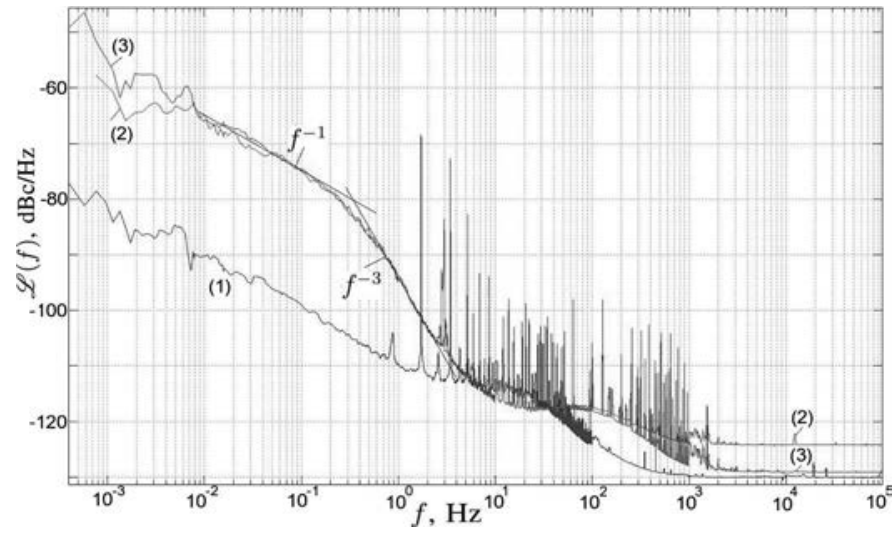

Fig. 8. Phase noise power spectral densities: (1) measurement phase noise floor, (2) cryogenic part phase noise for the 5th overtone of the Amode with $-4.5 \mathrm{dBm}$ synthesizer output signal, (3) cryogenic part phase noise for the 5 th overtone of the A-mode with $+0.5 \mathrm{dBm}$ synthesizer output signal.

main difference between the obtained results is seen in the region of high Fourier frequencies. As expected, the white noise of the experiment is significantly lower for the higher excitation level, resulting from a better signal-to-noise ratio. In all other ways, the results follow the same trend: no significant change is found for the crystal flicker noise.

Another experiment is made with the resonator working for different amounts of time in cryogenic conditions. As before, two cases are considered. The first measurement is made on the same day the cryocooler is turned on. This means that less than 12 hours elapsed between the time when the cryogenerator is turned on and the measurements. Nevertheless, the steady state seems to be reached according to the measured temperature. The second measurement is made after at least $24 \mathrm{~h}$ of cryogenerator permanent operation. The results are shown in Fig. 9 for the 5 th overtone of the A-mode and in Fig. 10 for the 3rd overtone of the same mode.

As seen in Fig. 9, there is no clear difference between two measurements separated in time. Both spectra have similar patterns and almost the same level.

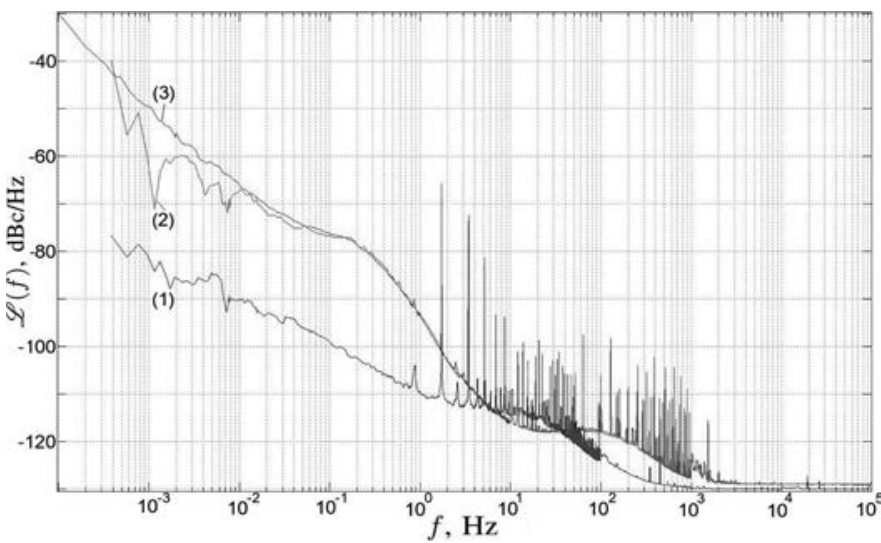

Fig. 9. Phase noise power spectral densities: (1) measurement phase noise floor, (2) cryogenic part phase noise for the A-mode 5th overtone measured within the first $12 \mathrm{~h}$ of cryogenerator operation, (3) cryogenic part phase noise for the A-mode 5 th overtone after at least $24 \mathrm{~h}$ of cryogenerator operation.

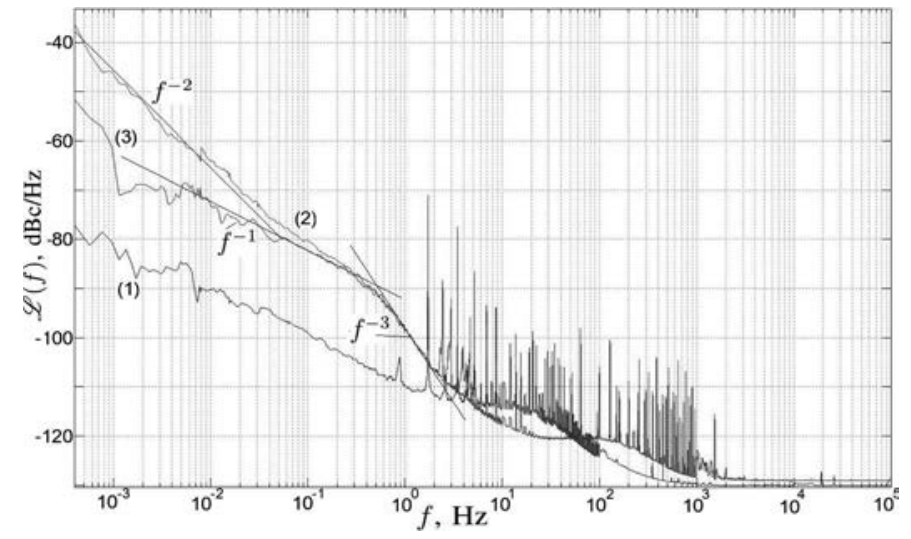

Fig. 10. Phase noise power spectral densities: (1) measurement phase noise floor, (2) cryogenic part phase noise for the 3rd overtone the Amode measured within the first $12 \mathrm{~h}$ of cryogenerator operation, (3) cryogenic part phase noise for the 3rd overtone of the A-mode after at least $24 \mathrm{~h}$ of cryogenerator operation.

For the time-separated measurements made for the 3rd overtone of the A-mode (see Fig. 10), a difference can be observed for low Fourier frequencies. The first-day measurement has a very short region of $f^{-1}$ slope, because the $f^{-2}$ noise is relatively high. In contrast, the second day measurements [Fig. 10, curve (3)] have a much less important $f^{-2}$ region. Because this type of noise depends on the temperature fluctuations and the temperature sensitivity of the mode, it can be concluded that the whole thermo-mechanical system (cryogenerator, cables, DUT, etc.) has some remaining temperature transient processes with time constants greater than $12 \mathrm{~h}$. In the same time, flicker noises of all the modes and overtones do not change with the amount of time of cryogenerator operation. To separate the influences of temperature fluctuations from vibration-caused noise of the resonator, the phase noise is measured for two different crystal device orientations and different temperatures. Fig. 11 presents the resonator phase noise when the resonator disk is vertical (i.e., the resonator disk is normal to the $y$-axis, according to Fig. 


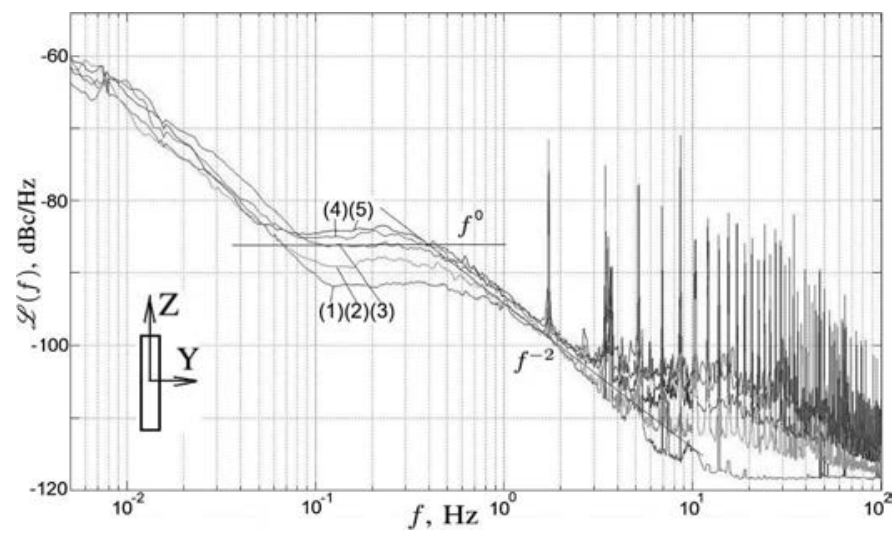

Fig. 11. Phase noise power spectral densities of the resonator (the 5th overtone of the A mode) installed vertically at different operating temperatures: (1) $5.3 \mathrm{~K},(2) 6 \mathrm{~K},(3) 7 \mathrm{~K}$, (4) $8 \mathrm{~K},(5) 9 \mathrm{~K}$.

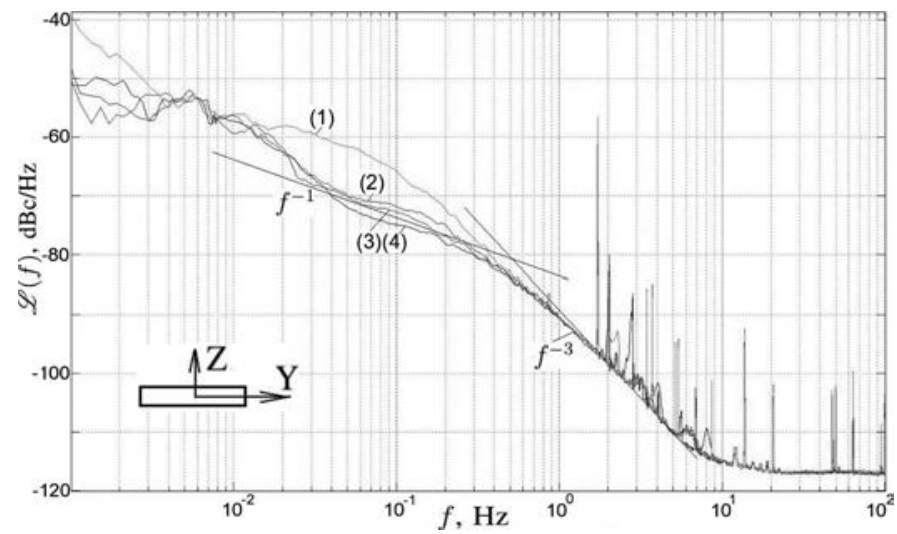

Fig. 12. Phase noise power spectral densities of the resonator (the 5th overtone of the A mode) installed horizontally: (1) $5.3 \mathrm{~K}$, (2) $6 \mathrm{~K}$, (3) $7 \mathrm{~K}$, (4) $8 \mathrm{~K}$.

1), whereas in Fig. 12 the resonator is horizontal (resonator disk normal to the $Z$-axis).

Taking into account the measured environmental disturbances (see Section II), comparison of Fig. 11 and 12 suggests the following conclusions:

1) temperature instabilities and vibrations are the main causes of the resonator phase noise when the cryocooler is operating;

2 ) as is the case at the usual temperatures, the resonator phase noise is shaped with a low-pass transfer function in the phase domain [21], [22], [23] with $f^{0}$ and $f^{-1}$ fluctuations at the input (see Fig. 13);

3) levels of $f^{0}$ and $f^{-1}$ fluctuations depend on crystal orientation and temperature sensitivity at a given temperature;

4) vibrations of the crystal with a $f^{-1}$ PSD result in a resonator $f^{-1}$ phase noise (in other words, the resonator flicker phase noise is due to external vibrations);

5) the quartz crystal resonator is most sensitive to vibration when the main vibration axis is normal to the resonator plate (this is an obvious mechanism, in fact);

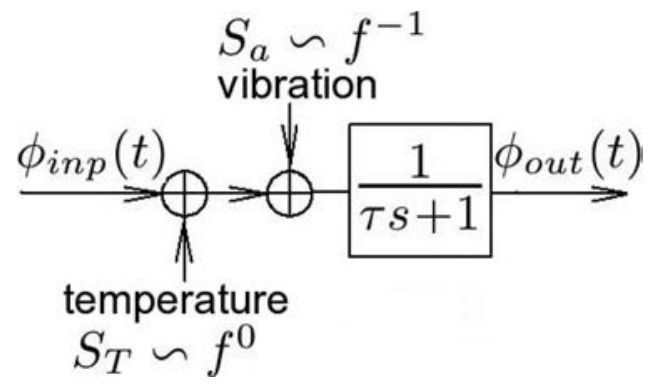

Fig. 13. Model of the resonator phase noise at cryogenic temperatures. $\tau$ is the resonator relaxation time $\tau=2 Q_{1} / \omega_{0} . \phi_{\text {inp }}(t)$ and $\phi_{\text {out }}(t)$ are the input and output phase fluctuations, respectively.

6) temperature instabilities of the crystal with a $f^{0}$ PSD result in a resonator $f^{0}$ phase noise;

7) temperature sensitivity of quartz crystal resonators decreases with temperature (this fact agrees with direct measurements of resonator characteristics [15]).

As a general conclusion to these points, it could be stated that the influence of the vibration on resonator parameters and noise can be reduced simply by choosing the proper angle of resonator orientation relative to the main (vertical) axis of vibration.

Following the same procedure as in Section III, an oscillator frequency stability floor can be estimated. This time it is supposed that the $f^{-1}$-like resonator noise is hidden by its $f^{0}$ noise caused by temperature in the $5.3 \mathrm{~K}$ measurement case [Fig. 11, curve (1)]. The maximum level of such a noise would have a power spectral density of $\mathcal{L}(f) \approx$ $-102 \mathrm{dBc} / \mathrm{Hz}$ in the worst case. This value gives an Allan deviation floor of the oscillator of $\sigma_{y}=4.2 \cdot 10^{-13}$ for $Q_{1}$ $=15.6 \cdot 10^{6}$. Even in this case, the oscillator frequency stability is not better than that of the state-of-the-art room-temperature quartz oscillators.

The measurement capability of the presented approach is limited by the test bench flicker noise $[\mathcal{L}(f)=-110 \mathrm{dBc} /$ $\mathrm{Hz}$ at $f=1 \mathrm{~Hz}]$. Therefore, the corresponding measurement noise floor of potential oscillators is also limited by $\sigma_{y}=1.7 \cdot 10^{-13}$ for the same value of the loaded quality factor $Q_{1}=15.6 \cdot 10^{6}$.

\section{COMparison With Earlier Works}

Three works give values of the flicker noise for resonators operating in cryostats [7], [10], [11]. Note that all three works present values of the fractional frequency fluctuations $S_{y}(f)$ for just one value of the Fourier frequency $f=1 \mathrm{~Hz}$, and not over a wide Fourier frequency range, as in Figs. 7-10. However, all authors attribute these single values to the resonator flicker noise without any discussion of the shape of the phase noise PSD. In fact, as it is seen from the experimental curves, this frequency always belongs to the $f^{-3}$ spectrum region of the resonator noise. As can be seen, this situation is typical for cryogenic conditions because of the higher values of the quality factors 
and, thus, much lower resonator bandwidth. Therefore, for cryogenic conditions, the $1-\mathrm{Hz}$ Fourier frequency is most likely situated outside the resonator bandwidth, and for the room temperature it is usually inside with present-day technology. This could lead to unjustified lower values of the resonator flicker noise. Moreover, depending on the quality of the temperature control (this is an issue for cryostats) and the actual resonator temperature sensitivity, this frequency range can lie within the $f^{-2}$ phase noise slope, which is attributed to the random-walk temperature fluctuations. In this case, there is a risk of measuring only the temperature characteristics of the resonator-cryostat system, but not the resonator flicker noise.

Robichon et al. [7] reports more than a one order of magnitude decrease in the phase noise for an AT-cut crystal, going from room temperature $\left[S_{y}(1 \mathrm{~Hz})=1.6 \cdot 10^{-24}\right]$ to $4.2 \mathrm{~K}\left[S_{y}(1 \mathrm{~Hz})=1.4 \cdot 10^{-25}\right]$. The authors do not give any theoretical explanation for this phenomenon, stating only correlation with the increase of the unloaded $Q$.

Similar results are given by Gagnepain et al. [10]. This work attempts to relate the resonator $1 / f$ noise and the quantum $1 / Q^{4}$ law [24], [25], which is itself questionable [26], [27], [28]. The authors admit the deviation of the measurement results from the theoretical law and make a guess that this fact is attributed to the presence of different loss phenomena and the dependence of the resonator parameters on temperature.

The work of Planat and Gagnepain [11] presents the dependence of the resonator fractional frequency fluctuations $S_{y}$ at $1 \mathrm{~Hz}$ on the temperature between $4 \mathrm{~K}$ and $30 \mathrm{~K}$. The work reports the strong correlation between acoustic wave attenuation around a phonon-phonon relaxation peak $(20 \mathrm{~K})$ and the $1 / f$ noise. In fact, the results clearly show the absence of a connection between measured noise and the $Q^{-4}$ law. The phase noise at $1 \mathrm{~Hz}$ from the carrier exhibits a considerable increase for temperatures below 7K. As previously mentioned, this may be attributed to difficulties with temperature regulation at very low temperatures. According to this work, $S_{y}$ at $1 \mathrm{~Hz}$ equals about $1.5 \cdot 10^{-20}$ for the same type of $5-\mathrm{MHz}$ AT-cut resonators. Thus, this result is almost 5 orders of magnitude higher than that shown in [7], [10].

Therefore, the previous works do not give an irrefutable answer on the question of the nature and even the value of the resonator flicker noise at liquid helium temperatures.

\section{CONCLUSION}

In this work, the resonator phase noise at cryogenic conditions is studied as a function of different parameters. The origins of the excess noise are determined. It is shown that vibration-caused noise could be significantly reduced by an appropriate choice of the device orientation in relation to the cryogenerator's vibration axes. Therefore, temperature instabilities remain the main source of the device phase noise. Estimations suggest that an oscillator based on such resonators working in such conditions can- not outperform state-of the-art room-temperature quartz oscillators. Because it was found that the main limitations result from temperature instabilities, two solutions could be proposed. First, the temperature control can be improved. The second solution is to find a crystal cut with a turn-over point in the temperature-frequency characteristic for liquid helium temperatures because the existence of such cuts is not known. Though FC-cut resonators [6], [7] already exhibit a turn-over point, its parameters are not satisfactory for temperature compensation (it has a narrow parabolic shape rather than the desired cubic behavior). To find a satisfactory cut, the parameters of quartz at deep cryogenic temperatures must be derived. The identification of the flicker noise source can be a clue to the same problem at room temperature.

\section{ACKNOWLEDGMENTS}

Special thanks are given to the staff of the Time and Frequency Department of FEMTO-ST Institute, Besançon, France, for their help and fruitful discussions.

\section{REFERENCES}

[1] H. E. Bömmel, W. P. Mason, and A. W. Warner, "Dislocations, relaxations and anelasticity of quartz crystal quartz," Phys. Rev., vol. 102 , no. 1 , pp. $64-71,1955$.

[2] W. P. Mason, "Use of internal friction measurements in determining the causes of frequency instabilities in mechanical vibrating frequency standards," IRE Trans. Instrum., vol. I-7, no. 3, pp. 189-200, 1958.

[3] A. W. Warner, "Ultra-precise quartz crystal frequency standards," IRE Trans. Instrum., vol. I-7, no. 3, pp. 185-188, 1958.

[4] N. Smagin, "Quartz crystal resonators with quality factors of about $120 \cdot 10^{6}$ at $2 \mathrm{~K}$," Izmeritel Tekh. SSSR, no. 151, 1960.

[5] G. Mossuz, "Study and achievement of a quartz crystal oscillator at very low temperature," Ph.D. thesis, Time and Frequency Dept., Université de Franche-Comté, Besançon, France, Jul. 1975.

[6] J. J. Suter, "Acoustic loss phenomena in alpha quartz resonators over the 1.4-77K temperature range," J. Appl. Phys., vol. 63, no. 11, pp. 5594-5595, Jun. 1988.

[7] G. Robichon, J. Groslambert, and J. J. Gagnepain, "Frequency stability of quartz crystal at very low temperatures: Preliminary results," in Proc. 38th Annu. Frequency Control Symp., Philadelphia, PA, 1984, pp. 201-205.

[8] A. El Habti, "Study of resonators and oscillators at very low temperatures," Ph.D. thesis, Time and Frequency Dept., Université de Franche-Comté, Besançon, France, Jan. 1993.

[9] A. El Habti and F. Bastien, "Low temperature limitation on the quality factor of quartz resonators," IEEE Trans. Ultrason. Ferroelectr. Freq. Control, vol. 41, no. 2, pp. 250-255, 1994.

[10] J. J. Gagnepain, J. Uebersfeld, G. Goujon, and P. Handel, "Relation between $1 / f$ noise and $Q$-factor in quartz resonators at room and low temperatures, first theoretical interpretation," in Proc. 35th Annu. Frequency Control Symp., 1981, pp. 476-483.

[11] M. Planat and J. J. Gagnepain, " $1 / f$ noise in quartz crystal resonators in relation with acoustic losses and frequency dispersion," Appl. Phys. Lett., vol. 50, no. 9, pp. 510-512, Mar. 1987.

[12] S. Galliou, J. Imbaud, R. Bourquin, N. Bazin, and P. Abbé, "Outstanding quality factors of bulk acoustic wave resonators at cryogenic temperature," in Proc. European Frequency and Time Forum 2008, [CD-ROM] Toulouse, France.

[13] S. Galliou, J. Imbaud, R. Bourquin, N. Bazin, and Ph. Abbé, "Quartz crystal resonators exhibiting extremely high Q-factors at cryogenic temperatures," Electron. Lett., vol. 44, no. 14, pp. 889 890,2008 
[14] S. Galliou, J. Imbaud, M. Goryachev, R. Bourquin, and Ph. Abbé, "Losses in high quality quartz crystal resonators at cryogenic temperatures," Appl. Phys. Lett., vol. 98, no. 9, art. no. 091911, 2011.

[15] M. Goryachev, S. Galliou, J. Imbaud, R. Bourquin, and Ph. Abbé, "Recent investigations on BAW resonators at cryogenic temperatures," in Proc. IEEE Int. Frequency Control Symp./European Frequency and Time Forum 2011, pp. 1-6.

[16] R. J. Besson, "A new electrodeless resonator design," Proc. 31st Annu. Frequency Control Symp., 1977, pp. 147-152.

[17] E. P. Eernisse, "Quartz resonator frequency shifts arising from electrode stress," Proc. 29th Annu. Frequency Control Symp., 1975, pp. $1-4$.

[18] E. Rubiola, J. Groslambert, M. Brunet, and V. Giordano, "Flicker noise measurement of HF quartz resonators," IEEE Trans. Ultrason. Ferroelectr. Freq. Control, vol. 47, no. 2, pp. 361-368, Mar. 2000.

[19] J. J. Gagnepain, M. Oliver, and F. L. Walls, "Excess noise in quartz crystal resonators," in 37th Annu. Symp. Frequency Control, 1983, pp. 218-225.

[20] R. Bourquin, D. Nassour, and D. Hauden, "Amplitude frequency effect of SC-cut quartz trapped energy resonators," in Proc. 36th Annu. Freq. Contr. Symp., 1981, pp. 200-207.

[21] F. Sthal, S. Galliou, J. Imbaud, X. Vacheret, P. Salzenstein, E. Rubiola, and G. Cibiel, "About quartz crystal resonator noise: Recent study," in 20th Int. Conf. Noise and Fluctuations, Pisa, Italy, Jun. 2007, pp. 607-610.

[22] E. Rubiola, Phase Noise and Frequency Stability in Oscillators. New York, NY: Cambridge University Press, Nov. 2008.

[23] E. Rubiola and V. Giordano, "On the $1 / f$ frequency noise in ultrastable quartz oscillators," IEEE Trans. Ultrason. Ferroelectr. Freq. Control, vol. 54, no. 1, pp. 15-22, Jan. 2007.

[24] P. H. Handel, "Quantum approach to $1 / f$ noise," Phys. Rev. A, vol. 22 , no. 2, pp. $745-757,1980$.

[25] P. H. Handel, "Nature of $1 / f$ frequency fluctuation in quartz crystal resonators," Solid-State Electron., vol. 22, no. 10, pp. 875-876, 1979.

[26] P. Dutta and P. M. Horn, "Low-frequency fluctuations in solids: $1 / f$ noise," Rev. Mod. Phys., vol. 53, no. 3, pp. 497-516, 1981.

[27] L. B. Kiss and P. Heszler, "An exact proof of the invalidity of 'Handel's quantum $1 / f$ noise model', based on quantum electrodynamics," J. Phys. C, vol. 19, no. 27, pp. L631-L633, 1986.

[28] Th. M. Nieuwenhuizen, D. Frenkel, and N. G. van Kampen, "Objections to Handel's quantum theory of $1 / f$-noise," Phys. Rev. A, vol. 35 , no. 6 , pp. 2750-2753, 1987. 